\title{
The LPM effect for EeV hadronic showers in ice: implications for radio detection of neutrinos
}

\author{
J. Alvarez-Muñiz and E. Zas. \\ Departamento de Física de Partículas, Universidade de Santiago \\ E-15706 Santiago de Compostela, Spain
}

\begin{abstract}
We study the longitudinal development of hadronic showers in ice for energies up to $100 \mathrm{EeV}$ and its implications for radio and optical Cherenkov emission. A small fraction of showers induced by primaries of energy above $1 \mathrm{EeV}$ is shown to display the characteristic elongation associated to the Landau Pomeranchuck Migdal (LPM) effect. The rest look like ordinary showers because the highest energy $\pi^{0}$ 's interact instead of decaying in two photons. The LPM effect observed in this fraction of the showers is mainly due to $\eta$ and $\eta^{\prime}$ production and decay. We give parameterizations for the total and excess charge tracklengths and for the amplitude and angular spread of the electric field spectrum in the Cherenkov direction. Implications for neutrino detection are briefly addressed.
\end{abstract}

PACS number(s): 96.40.Pq; 29.40.-n; 96.40.Kk; 96.40.Tv

Keywords: Cherenkov Radiation; LPM effect; Hadronic showers; Neutrino detection. 


\section{INTRODUCTION}

The detection of low energy neutrinos (MeV-GeV) from the Sun and from cosmic ray interactions in the atmosphere is now routine in underground detectors which we hope will reveal new aspects of the neutrino sector in the Standard Model [1]. On the other hand the wealth of information obtained with the detection of few $\mathrm{MeV}$ neutrinos from supernova SN1987A showed that the enormous potential of neutrino astronomy could be reality in the

near future. Several experiments are being constructed at present to detect neutrinos at higher energies, at which the atmospheric neutrino flux is expected to be sufficiently low to obtain new information from extraterrestrial sources. They were conceived to measure the Cherenkov light emitted by upcoming muons produced in charged current neutrino interactions that cross the Earth. The long muon ranges allow an effective volume sufficiently large to answer important questions, for example on the intriguing acceleration mechanisms in Active Galactic Nuclei (AGN) and Gamma Ray Bursts (GRB) [2].

It is becoming increasingly apparent that the highest energy neutrinos will play an important role. The identification of the highest energy gamma ray sources with Blazars, which are interpretated as AGN with jets pointing in our direction, has shifted theorists to model acceleration in the jets. These jets are believed to be particle flows with relativistic bulk motion and high associated Lorentz factors which naturally make the emission highly anisotropic and boost it to very high energies [3]. It is also believed that the infrared background prevents the $\mathrm{TeV}$ gamma ray emission from reaching the Earth except for a few nearby sources [4]. The possibility is open that particles are accelerated to much higher energies getting absorbed in the different backgrounds. Similarly for GRB's there are acceleration models reaching up to energies much greater than observed. Both phenomena are candidates for the intriguing origin of the highest energy cosmic rays [5],6]. If this is the case, large fluxes of neutrinos can be expected up to close to the highest energies the hadrons are accelerated. In any case EeV neutrinos must exist because of the interactions of cosmic rays with the microwave photons and their search can answer important issues 
with respect to these objects or other potential neutrino sources as well as to the origin of the highest energy cosmic rays.

EeV neutrinos have a high enough cross section that prevents them from traversing the Earth without interacting. These high energy neutrinos produce extremely high energy showers whose Cherenkov light may also allow efficient detection in the underground detectors in project or construction. Alternative detection mechanisms become favourable for extremely energetic showers. The radio technique relies on the coherent emission of Cherenkov light in radio waves by all shower particles when the wavelength of the emission is larger than the shower dimensions [7]. Ice is being considered as a good possibility because of the long attenuation length for radio waves [8]. Similarly the proposed acoustic alternative relies on detecting the coherent sound wave which is produced by the shower in ocean water [9]. The coherence condition makes the signals scale as the square of the incident particle energy and this is the reason why these methods become most promising at the highest energies. Depending on the actual fluxes the construction of large enough detectors sensitive to the expected fluxes may be cost effective provided these alternatives are succesfully implemented [10]. Several estimates of the neutrino expected event rates in these detectors have been made [11][3]. An interesting possibility is the detection of the radio pulses from cosmic ray and neutrino interactions on the Moon surface using radiotelescopes on Earth [14]. EeV shower behavior in dense media is important for EeV neutrino detection, particularly in water (or ice) as chose for most of the proposed detectors.

Above PeV energies the cross sections for pair production and bremsstrahlung get suppressed because the characteristic length of the interaction becomes larger than atomic spacing and collective effects of the atomic fields have to be considered. This is the Landau Pomeranchuck Migdal (LPM) effect [15]. Electromagnetic EeV showers have been shown to have huge elongations because of the LPM effect 16 18 and hadronic showers are expected to behave similarly if they contain $\mathrm{PeV}$ photons or electrons.

Here we study the development of EeV hadronic showers in water concentrating on the relevant parameters involved in the emission of Cherenkov light and coherent radio pulses. 
We will use many results presented in the study of electromagnetic showers from the same perspective as described in ref [18]. As it was the case for electromagnetic showers, the full simulation of Cherenkov light and in particular coherent effects between different particles is not practical because of the high energies involved. We choose to approximate shower development by a combination of Monte Carlo methods and parameterizations of showers of lower energies. The main characteristics of the radio pulse emission from these showers at the Cherenkov angle can be deduced from the longitudinal shower development so we will use one dimensional approximations which will only be valid for emission in this direction. Although more detailed calculations will be necessary, many of the most important aspects of the possibilities of detecting hadronic showers as induced by neutrino interactions can be answered with the approximations made here. Many of the qualitative conclusions obtained in this work can be easily extended to other media.

\section{HADRONIC SHOWERS.}

Shower development has been studied in air up to $10^{21} \mathrm{eV}$ because all high energy cosmic ray experiments look for them. At lower energies $(<1 \mathrm{TeV})$ they have also been studied in dense media for accelerator experiments. The study of purely electromagnetic showers and LPM implications, has been done in water for energies up to $10 \mathrm{EeV}$ [18. Such showers are produced at the lepton vertex in charged current Deep Inelastic Scattering (DIS) electron neutrino interactions. In these interactions the average energy transfer to the lepton is predicted to be large, about $75 \%$ [19].

Hadronic showers produced in both charged and neutral current DIS neutrino interactions are started by hadrons in the fragmentation of the nuclear debris. At high energies the multiplicity is expected to be about half of that in $e^{+} e^{-}$collisions for a center of mass energy squared equal to minus the square of the 4 -momentum transfer $\left(Q^{2}=-q^{\mu} q_{\mu}\right)$ in the DIS process [20]. Practically all models for neutrino production involve the decay of pions which naturally predict more muon flavor neutrinos from naive channel counting. The 
ratio of muon to electron neutrino production may be actually larger than expected in some environments because of muon synchrotron losses as has been recently stressed [21]. All these factors may well compensate the fact that in DIS interactions only one fourth of the neutrino energy is typically transferred to the hadron vertex. Multiple hadrons are also produced in $68 \%$ of the $W$-boson decay in the resonant electron antineutrino scattering with atomic electrons. Hadronic showers will also play an important role in tau neutrino detection through the proposed double bang events [22].

For EeV neutrinos the fragmented particles, mostly pions, will mantain the direction of the primary neutrino in the lab frame because their average transverse momentum is expected to be in the few hundred $\mathrm{MeV}$ range. These particles continue to interact with nucleons and they still have sufficient energy so that after three or four generations the cummulative angular deviations are still very small. The hadronic shower can be thought as having a hard penetrating central core containing mostly pions which feeds electromagnetic subshowers fundamentally through $\pi^{0}$ decay in two photons. Because the medium is dense, charged pions are expected to interact before decaying. Assuming energy equipartition between all flavor pions, a fraction of $1 / 3$ would go into electromagnetic subshowers every time there is an interaction. If all interactions occur simultaneously every interaction length (to make things simple) it is easy to find that after three generations $80 \%$ of the energy is in electromagnetic subshowers. The shower will have a markedly electromagnetic character because of the high energy involved and the medium density which prevents decays of most of the charged pions. The lateral distribution should be related to a sum of distributions of electromagnetic showers started at different depths. Near the core the showers are thus younger, dominated by small electromagnetic showers initiated deeper, while away from the shower axis they are due to early electromagnetic subshowers of higher energies.

Continuing with this simple model, we can consider that after a neutrino interaction with pion multiplicity $N$, the pion energy is roughly the energy transferred to the hadron $y E$ divided by $N$. The LPM effect is known to increase the electron and photon interaction 
lengths above some threshold energy $\sim E_{L P M}(2 \mathrm{PeV}$ for ice $)$ [17, 16,23]. However the longitudinal profile of electromagnetic showers in ice around shower maximum starts to be affected typically at primary energies above $E=20 \mathrm{PeV}$ [18]. For a $1 \mathrm{EeV}$ energy transfer to the hadron a multiplicity of order 15 can be expected in the $\nu$ interaction and the photons from the $\pi^{0}$ decays would roughly have $30 \mathrm{PeV}$. Moreover most of the electromagnetic energy in the shower is produced after the first generation. As a result little deviations from typical shower development are expected below 1 EeV because of the LPM effect.

As the primary energy rises so will the energy of the first generation $\pi^{0}$ 's. However they do not necesarily produce high energy photons. Neutral pions have a decay length $c \tau=2.510^{-6} \mathrm{~cm}$ and an interaction depth of about $130 \mathrm{gcm}^{-2}$ so that in the competition between decay and interaction the latter dominates for $\pi^{0}$ energies above about $6.7 \mathrm{PeV}$. As a result the showers can be expected to show LPM effects in a mitigated form. Even some 100 EeV showers are actually shown below to display small LPM elongations. EeV hadronic showers can look more like rescaled versions of lower energy showers while EeV electromagnetic showers are greatly distorted and elongated because of the LPM effect.

\section{SIMULATIONS.}

A complete simulation of the radio pulse should monitor particles down to the $\mathrm{MeV}$ range requiring unreasonably large times for EeV energies [24]. To investigate EeV hadronic shower characteristics we have developed a fast hybrid Monte Carlo which simulates 1 dimensional showers down to some crossover energy, at which the subshower produced is taken from a tested parameterization. For electromagnetic subshowers we use the Greisen parameterization which was shown in [24] to be valid up to energies of $100 \mathrm{TeV}$. We have calculated our own parameterizations for hadronic subshowers inititated by protons, pions and kaons. The code has been designed to calculate quantities such as the projected tracklength (onto

\footnotetext{
${ }^{*}$ Corresponds to the definition in Ref. 17.
} 
shower axis) and the weighted projected tracklength (excess tracklength due to electrons). These quantities are respectively known to be the relevant parameters for optical and radio Cherenkov emission [24]. We have used parameterizations of these tracklengths for electromagnetic showers using the results obtained in [24]. Intermediate results will be presented elsewhere.

The shower simulation part is based on UNICAS 25] which has been adapted to run in a homogeneous water medium with density $0.924 \mathrm{gcm}^{-3}$. The code has also been modifyed to take into account interactions of $\pi^{0}$ 's and other shortlived resonances as well as corrections to bremsstrahlung and pair production accounting for the Landau-Pomeranchuk-Migdal effect. For the lower energy showers we have performed full simulations. Above $100 \mathrm{TeV}$ we use the hadronic code SIBYLL [26] together with the parameterizations. SIBYLL includes "minijet events" which are responsible for the cross section increase with energy [27] and soft collisons based on the Dual Parton Model (DPM) [28].

We have made extensive independent checks of the codes used. For example full 1-D simulations (for energies below $100 \mathrm{TeV}$ ) are well within the uncertainties associated to the different hadronic models compared with CORSIKA in [29]. We have also checked the 1-D version of UNICAS in ice comparing photon showers with those obtained in the 3-D electromagnetic code developed specifically for calculating radio pulses in ice [24]. We have checked the hybrid approach both comparing it to full simulations and ensuring that all hybrid codes are stable under changes of the energy crossover.

\section{RESULTS.}

We have simulated showers initiated by protons, and most of the results presented here correspond to such showers. Little difference is observed between proton and pion initiated showers of the same primary energy. We have explored the energy range from $10 \mathrm{GeV}$ to 100 $\mathrm{EeV}$ simulating large numbers of proton showers (over 20,000 showers above $1 \mathrm{EeV}$ ) with

the described codes. The lower energy showers are necessary to make parameterizations 
which allow the simulation of the highest energy ones.

We firstly show the results of the fraction of energy going into electromagnetic showers in Fig. 1. Here the fraction is seen to rise with energy according to the simplified model above, until the effect of neutral pion interactions becomes important. In the total tracklength calculation, relevant for the Cherenkov light output, the electromagnetic contribution accurately scales with the electromagnetic energy in the shower as shown in Fig. 1. Other charged particles contribute some percentage which decreases with energy (15\% at most) as can be expected from the electromagnetic energy fraction in Fig. 1. The coherent radio pulse emission scales with the difference between positive and negative charge tracklengths, the weighted tracklength, to which particles other than electrons and positrons contribute negligibly. We also display "shower length" in Fig. 1, defined in [18] as the length along which shower size exceeds $70 \%$ of its maximum. The angular width of the Cherenkov radiopulse emitted at the Cherenkov angle is approximately inversely proportional to this parameter.

Hadronic and electromagnetic showers have well known differences which have been studied at high energies only in air, mainly hadronic showers have larger attenuation length", reach deeper from first interaction point, have harder lateral distributions and carry a significant fraction of their energy in muons and neutrinos. As a result they have slightly less particles at maximum than electromagnetic showers of the same primary energy. These differences can be seen in Fig. 2 where proton showers are compared to pure electromagnetic showers of energies $10 \mathrm{TeV}, 1$ and $100 \mathrm{PeV}$. As long as the LPM effect is not significant, the showers "scale" with primary energy in much the same way as electromagnetic showers, what incidentally, simplifies their parameterizations.

Below $1 \mathrm{EeV}$ the longitudinal profile of hadronic showers has this scaling behavior, characterisitc of showers that are not affected by the LPM effect. This is not surprising, the

\footnotetext{
$\dagger$ Attenuation length is defined as the length over which the energy drops by a factor $e$ in the region well after shower maximum and it is proportional to the particle interaction length.
} 
average energy of the pions is well below the primary interaction energy because of the large multiplicities involved [30] and only electromagnetic showers of energy above $\sim 20 \mathrm{PeV}$ show "scaling deviations" [18]. In table I we give the mean energy of the $\pi^{0}$ 's as a function of primary energy as obtained in the hadronic interaction generator. In Fig. 3 we compare the energy distributions of all the first generation $\pi^{0}$ s in proton-ice interactions at different energies to those that actually decay into photons. Only about $10 \%$ of the $\pi^{0}$ 's are expected to produce photons of energy above $20 \mathrm{PeV}$ because most of them interact. Showers are not elongated despite being induced by primaries exceeding $E_{L P M}$ by several orders of magnitude. In Fig. 1 we also display shower length neglecting neutral pion interactions to further illustrate this point.

In this respect the role of resonances with shorter lifetimes than the $\pi^{0}$ can be more important if they have decay channels involving electrons or photons. If these resonances are produced in early interactions, high energy photons (or electrons) produce subshowers with characteristic LPM elongations. The extent of this effect is a probabilistic issue and depends on detailed aspects of the hadronic interaction model. At the highest energies, experimental evidence is non-existant and our results have to be taken with caution. They indicate that these aspects are important for shower development and it may be that, if these showers are ever observed, they would provide experimental information on such channels. In particular we find that the $\eta$ and the $\eta^{\prime}$ contribute most and this is just because they are very short lived, they are produced relatively often and they have considerable branchings into photons, $\sim 40 \%(\sim 65 \%)$ for $\eta\left(\eta / \mathrm{f}^{\prime}\right)$.

We find that a proportion of showers above $1 \mathrm{EeV}$ have deep tails characteristic of LPM showers (see Fig. 4). We also note that the energy content of these tails (proportional to shower development integral) is typically of order $1 \%$. All of these can be quantitatively addressed with the aid of Fig. 5, representing the "leading photon" distribution as obtained

\footnotetext{
${ }^{\ddagger}$ Including the $\eta^{\prime} \rightarrow \eta \pi^{+} \pi^{-}$decay chain.
} 
in SYBILL, that is the differential probability that the highest energy photon in a shower has a fraction energy $\left(x=E_{\gamma} / E_{0}\right)$ in the range $(x, x+\mathrm{d} x)$. The median is at about $2 \%$ and not very sensitive to primary energy, hence about $50 \%$ of the hadronic showers having energy above $50 E_{t}$ are expected to have a photon of energy $>E_{t}$. If $E_{t}$ is chosen say above $100 \mathrm{PeV}$, the electromagnetic subshower generated by the leading photon will have a long LPM tail. The range of leading photon energy fractions is between $0.1 \%$ and $30 \%$ in $99.9 \%$ of the showers and one can easily deduce that it is practically impossible to have a shower without an LPM tail if the primary energy is above $100 \mathrm{EeV}$ and that hadronic showers of energy below $250 \mathrm{PeV}$ will hardly have LPM effects.

\section{CHERENKOV RADIATION FROM HADRONIC SHOWERS.}

Cherenkov light is emitted as charged particles travel through a medium at a speed greater than that of light. While the electric field amplitude is proportional to particle tracklength, in the optical region the emission is incoherent and the power output scales with the total tracklength [31]. For wavelengths exceeding the shower dimensions Cherenkov light is emitted coherently by all shower particles, opposite charges contribute with opposite phases and the electric field amplitude is proportional to the weighted tracklength [32]. The excess electrons in electromagnetic showers are caused by low energy interactions with atomic electrons 24,32 . The matter antimatter symmetry in the cross sections is broken by the absence of target positrons. The dominant process is Compton scattering with atomic electrons, but electron positron annihilation, Möller and Bhabha scattering also contribute to an overall $21 \%$ excess when expresssed in terms of the total tracklength [24]. Many of the results obtained in the context of radio emission from electromagnetic showers [18,24] can be applied to our hadronic shower simulation results.

There is a smooth transition from the incoherent to the coherent Cherenkov regions which has been described in [18,24]. It occurs in ice at $\sim 1 \mathrm{GHz}$ for radiation emitted in the Cherenkov angle direction and at lower frequencies away from this direction. The spectrum 
rises linearly with frequency until interference effects from different shower regions start to take place. This happens at a maximum frequency $\nu_{\max }$. For pulses observed at the Cherenkov angle the interference is governed by the lateral distribution, the narrower the lateral spread the bigger the maximum frequency. We expect it not to be too different from electromagnetic showers, and we take $\nu_{\max }$ from them. As a first approximation this is justified because most energy in hadronic showers is electromagnetic, and consists of much lower energy subshowers.

The electric field amplitude in the Cherenkov direction can be approximated quite accurately as a 1-D Fourier transform of the longitudinal charge excess distribution. The half width of the central peak is inversely proportional to shower length as defined above and shown in Fig. 1. This has been used to describe Cherenkov emission from EeV electromagnetic showers in ice [18]. Since the highest output signal is concentrated in the Cherenkov peak, its size and angular spread are the most relevant aspects of such radiation, particularly for establishing the detection capabilities of future detectors. These properties are calculable in this approach and can be used for detector design while more detailed simulations are implemented.

The change in shower length with energy is very small, $\sim 30 \%$ between $100 \mathrm{TeV}$ and $10 \mathrm{EeV}$ as shown in Fig. 1. As a result the angular distribution of the Fourier transform of the radiopulse is not going to become narrower for higher energies contrary to electromagnetic showers [18]. For those showers that do display LPM effects the narrowing can be observed in a rather convoluted diffraction pattern, see Fig. 6; the Cherenkov radiopulse being coherent contains all the information on the excess charge distribution. The lateral distributions of the excess charge also affects the full diffraction pattern behavior in other directions but this is not addressed in this work.

From our shower results it is straigthforward to obtain a parameterization for the total tracklength $t$ from which the total Cherenkov light in the optical band can be obtained:

$$
f(\epsilon)=-1.2710^{-2}-4.7610^{-2}(\epsilon+3)-2.0710^{-3}(\epsilon+3)^{2}+0.52 \sqrt{\epsilon+3}
$$




$$
\begin{gathered}
\epsilon=\log _{10} \frac{E_{0}}{1 \mathrm{TeV}} \\
t=6.25 f(\epsilon) \frac{E_{0}}{1 \mathrm{GeV}} \mathrm{m}
\end{gathered}
$$

The weighted projected tracklength is $\sim 0.21 t$ and the normalization of the radiopulse electric field spectrum at the Cherenkov peak $\vec{E}\left(\omega, R, \theta_{C}\right)$ scales with it:

$$
R\left|\vec{E}\left(\omega, R, \theta_{C}\right)\right|=1.1 \times 10^{-7} \mathrm{f}(\mathrm{z}) \frac{E_{0}}{1 \mathrm{TeV}} \frac{\nu}{\nu_{0}} \frac{1}{1+0.4\left(\frac{\nu}{\nu_{0}}\right)^{2}} \mathrm{~V} \mathrm{MHz}^{-1}
$$

where $\nu$ is the observation frequency and $\nu_{0} \approx 500 \mathrm{MHz}$.

Lastly the angular spread of the electric field is inversely proportional to shower length:

$$
\begin{gathered}
E(\omega, R, \theta)=E\left(\omega, R, \theta_{C}\right) \mathrm{e}^{-\ln 2\left[\frac{\theta-\theta_{C}}{\Delta \theta}\right]^{2}} \\
\Delta \theta \simeq\left\{\begin{array}{l}
1^{\mathrm{o}} \frac{\nu_{0}}{\nu}\left(2.07-0.33 \epsilon+7.510^{-2} \epsilon^{2}\right) \text { for } 1 \mathrm{TeV}<E_{0}<100 \mathrm{TeV} \\
1^{\circ} \frac{\nu_{0}}{\nu}\left(1.74-1.2110^{-2} \epsilon\right) \text { for } 100 \mathrm{TeV}<E_{0}<100 \mathrm{PeV} \\
1^{\mathrm{o}} \frac{\nu_{0}}{\nu}\left(4.23-0.785 \epsilon+5.510^{-2} \epsilon^{2}\right) \text { for } 100 \mathrm{PeV}<E_{0}<10 \mathrm{EeV}
\end{array}\right.
\end{gathered}
$$

\section{DISCUSSION.}

We have shown that EeV hadronic showers produced in neutrino interactions are very different from electromagnetic showers. The discussion above applies to showers initiated by protons or pions of a given primary energy. A $100 \mathrm{EeV}$ neutrino transferres $25 \%$ of its energy to the hadronic debris with typical multiplicity 17 and only the leading particle keeps a significant fraction $1-K$ of the primary neutrino energy, where $K$ is the mean inelasticiy. As a result practically all of the electromagnetic subshowers will have energies far removed from the neutrino energy. We can expect the hadronic showers induced by neutrinos up to $10^{3} \mathrm{EeV}$ to have a quite ordinary longitudinal development curves around shower maximum. They will however differ considerably from the electromagnetic showers produced in charged current electron neutrino interactions which display a typical fluctuating nature, reaching a great deal further and having many less particles at shower maximum [18]. 
It should be in principle possible to distinguish them comparing the shower profiles if they occur inside optical Cherenkov detectors such as those now in construction. For radiodetection the differences are converted to differences in the angular width around the Cherenkov direction. This effect favours the detection of hadronic induced pulses because the antenna spacing would not have to be reduced. It is not clear that at EeV energies the electron neutrino contributions for the electromagnetic pulses dominate. The answer depends crucially on the relative electron to muon neutrino fluxes, the fraction of energy transferred to the nucleons in DIS interactions and the relative spacing between antennas. With an array of antennas sufficiently dense it should be possible to recognize the much broader pulses for EeV hadronic showers. This is very interesting since the relative rates of each type can be related to the ratio of electron to muon neutrinos. This could be used to test neutrino mixing parameters models in regions of parameter space unaccesible to other experiments and similar to those suggested in Ref. [33].

Acknowledgements: We thank T. Stanev for making UNICAS available to us and for many discussions and suggestions on this work, we also thank R.A. Vázquez for useful comments and for carefully reading the manuscript and L.G. Dedenko, F. Halzen, J. Knapp, and P. Lipari for fruitful discussions. This work was supported in part by CICYT (AEN961673) and by Xunta de Galicia (XUGA-20604A96). One of the authors J. A. thanks the Xunta de Galicia for financial support. 


\section{REFERENCES}

[1] M.C. Gonzalez-García, H.Nunokawa, O.L.G. Peres, T.Stanev, J.W.F. Valle, e-print archive hep-ph 9801368 .

[2] T.K. Gaisser, F. Halzen, T. Stanev, Phys. Rep. 258 (1995) 173 and references therein.

[3] P. Padovani in Rencontres de Moriond: High-Energy Phenomena in Astrophysics, Les Arcs, France, 18-25 Jan 1997, e-Print Archive: astro-ph/9704002.

[4] F. W. Stecker, O. C. De Jager and M. H. Salamon, Astrophysical Journal 390, L49 (1992).

[5] Biermann, P.L. in Proc. of the ICRR International Symposium: Astrophysical Aspects of the most energetic Cosmic Rays (Kofu, Japan, November 1990), eds. M. Nagano and F. Takahara (World Scientific, 1991).

[6] E. Waxman, Phys. Rev. Lett. 75 (1995) 386-389.

[7] G.A. Askar'yan, Soviet Physics JETP 14,2 (1962) 441; 48 (1965) 988.

[8] M.A. Markov, I.M. Zheleznykh, Nucl. Instr. and Methods in Phys. Research A248 (1986) 242.

[9] J.G. Learned in Proc. of the Second Venice Workshop on Neutrino Telescopes; Venice (1990), ed. M. Baldo-Ceolin, Feb. 1990; L.G. Dedenko et al. e-print archive: astroph/8705189.

[10] J.V. Jelley, Astroparticle Physics 5 (1996), pp.255-261.

[11] G.M. Frichter, J.P. Ralston, D.W. Mc Kay, Phys. Rev. D 53 (1996) 1684.

[12] P.B. Price, Astroparticle Physics 5 (1996) 43.

[13] A.L. Provorov, I.M. Zheleznykh, Astroparticle Physics 4 (1995) 55.

[14] R.D. Dagkesamansky and I.M. Zheleznykh, Radioastronomical Method of the Neutrino 
and Hadron Detection, in Proc. of the ICRR International Symposium: Astrophysical Aspects of the most energetic Cosmic Rays (Kofu, Japan, November 1990), eds. M. Nagano and F. Takahara (World Scientific, 1991) p.373; J. Alvarez-Muñiz and E. Zas, in Proc. of XXVth International Cosmic Ray Conference, Durban, South Africa, Ed. M.S. Potgieter et al. (1997) Vol.7, pp. 309-312.

[15] L. Landau and I. Pomeranchuk, Dokl. Akad. Nauk SSSR 92 (1953) 535; 92 (1935) 735; A.B. Migdal, Phys. Rev. 103 (1956) 1811; Zh. Eksp. Teor. Fiz. 32 (1957) 633 [ Sov. Phys. JETP 5 (1957) 527].

[16] A. Misaki, Phys. Rev. D 40 (1989) 3086, Nucl. Phys. B (Proc. Suppl.) 33 (1993) 192-199 and related papers.

[17] T. Stanev, Ch Vankov, R.E. Streitmatter, R.W. Ellsworth and T. Bowen, Phys. Rev. D 25 (1982) 1291.

[18] J. Alvarez-Muñiz and E. Zas, Physics Letters B 411 218-224 (1997).

[19] R. Gandhi, C. Quigg, M.H. Reno and I. Sarcevic, Astroparticle Phys. 5 (1996) 81-110. [20] N. Schmitz, Int. Journal of Mod. Phys. A8 (1993) 1993.

[21] J.P. Rachen, P. Mészáros, submitted to Phys. Rev. D, e-print archive astro-ph/9802280.

[22] J.G. Learned and S. Pakvasa, Astropart. Phys. 3 (1995) 267

[23] For a comprehensible review on suppression of bremsstrahlung and pair production see S.R. Klein, e-Print Archive: hep-ph/9802442, to be published in Rev. Mod. Phys; S.R. Klein, e-Print Archive: astro-ph/9712198.

[24] F.Halzen, E.Zas, T.Stanev, Phys. Lett. B 257 (1991) 432; E.Zas, F.Halzen, T.Stanev, Phys. Rev. D 45 (1992) 362.

[25] T.K. Gaisser, Cosmic Rays and Particle Physics, Cambridge University Press 1990. 
[26] R.S. Fletcher, T.K. Gaisser, P. Lipari and T. Stanev, Physical Review D 50,9 (1990) p. 5710.

[27] D. Cline, F. Halzen and J. Luthe, Phys. Rev. Lett. 31 (1973) 491; F.Halzen, Nucl. Phys. B92 (1975) 404; F. Halzen and E. Zevgolatakos, Phys. Lett. B 93 (1980) 472; F. Halzen and F. Herzog, Phys. Rev. D 30 (1984) 2326; T.K. Gaisser and F. Halzen, Phys. Rev. Lett. 54 (1985) 1754.

[28] A. Capella, U. Sukhatme, C-I Tan and J. Tran Thanh Van Phys. Reports 236 (1994) 225-329.

[29] J.Knapp, D.Heck and G.Schatz, Preprint Forschungszentrum Karlsruhe, FZKA 5828, Draft from September 1996.

[30] R.M. Barnett et al., Review of Particle Physics, Phys. Rev. D 54, 1 (1996)

[31] I. Frank and I. Tamm, Dokl. Akad. Nauk SSSR 14, 109 (1937).

[32] H.R. Allan, Progress in Elementary Particles and Cosmic Ray Physics (North Holland, Amsterdam, 1971), Vol. 10, p. 171.

[33] F. Halzen and D. Saltzberg, e-print Archive astro-ph 9804354. 


\section{FIGURE CAPTIONS}

Figure 1: Left: Electromagnetic energy fraction as a function of primary energy (solid) and ratio of the weighted projected tracklength and shower energy in units of $\mathrm{km} \mathrm{TeV}^{-1}$. Right: Shower length (in radiation lengths and defined in text) in a proton shower as a function of primary energy. The solid (dashed) line represents shower length when competition between interaction and decay for $\pi^{0}$ 's is (is not) performed.

Figure 2: Shower development of a proton (solid) and electromagnetic (dashed) showers of primary energies $10 \mathrm{TeV}, 1 \mathrm{PeV}$ and $100 \mathrm{PeV}$.

Figure 3: Energy distribution of $\pi^{0}$ 's in $10 \mathrm{EeV}, 1 \mathrm{EeV}$ and $100 \mathrm{PeV}$ proton-ice interactions showing the average number of $\pi^{0}$ 's with energy greater than $x E_{\pi}\left(10^{4}\right.$ events). The three overlapping upper curves correspond to all the $\pi^{0}$ 's produced while the three lower curves count only those $\pi^{0}$ 's that decay.

Figure 4: Shower development of individual showers of energies $10 \mathrm{EeV}$ (solid histograms) and $1 \mathrm{EeV}$ (dashed lines). For $1 \mathrm{EeV}$ we also show the average over 100 showers (dotted line). Labels refer to the individual showers taken to obtain Fig. 6.

Figure 5: Leading photon distributions for $10 \mathrm{EeV}$ and $100 \mathrm{EeV}$ hadronic showers. The area under this graph, as shown, is proportional to the probability.

Figure 6: Radiopulse distribution around the Cherenkov direction for the two showers labelled in Fig. 4, illustrating the effect of the LPM tail on the radiopulse angular distribution.

TABLES

\begin{tabular}{|c|c|c|c|c|}
\hline$E_{0}(\mathrm{TeV})$ & $10^{4}$ & $10^{5}$ & $10^{6}$ & $10^{7}$ \\
\hline$<E_{\pi^{0}}>(\mathrm{TeV})$ & 40 & 300 & $210^{3}$ & $1.710^{4}$ \\
\hline
\end{tabular}

Table I: Average neutral pion energy in proton proton collisions for different projectile energies in the lab frame as produced by SIBYLL hadronic generator. 
$\mathrm{x} / \mathrm{N} \mathrm{dN} / \mathrm{dx}$

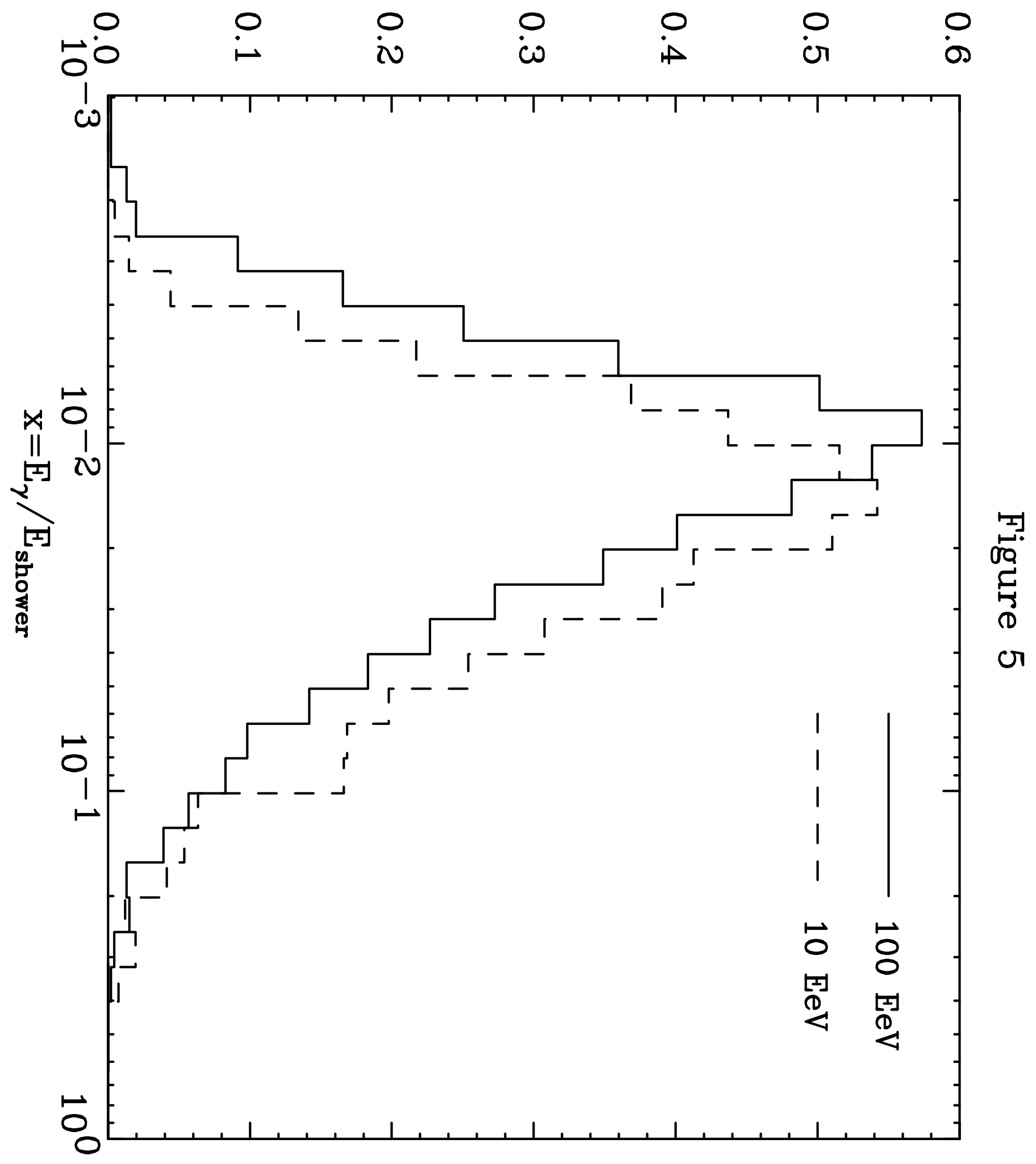


$\operatorname{Mod}(E(\omega))$ (arbitrary units)

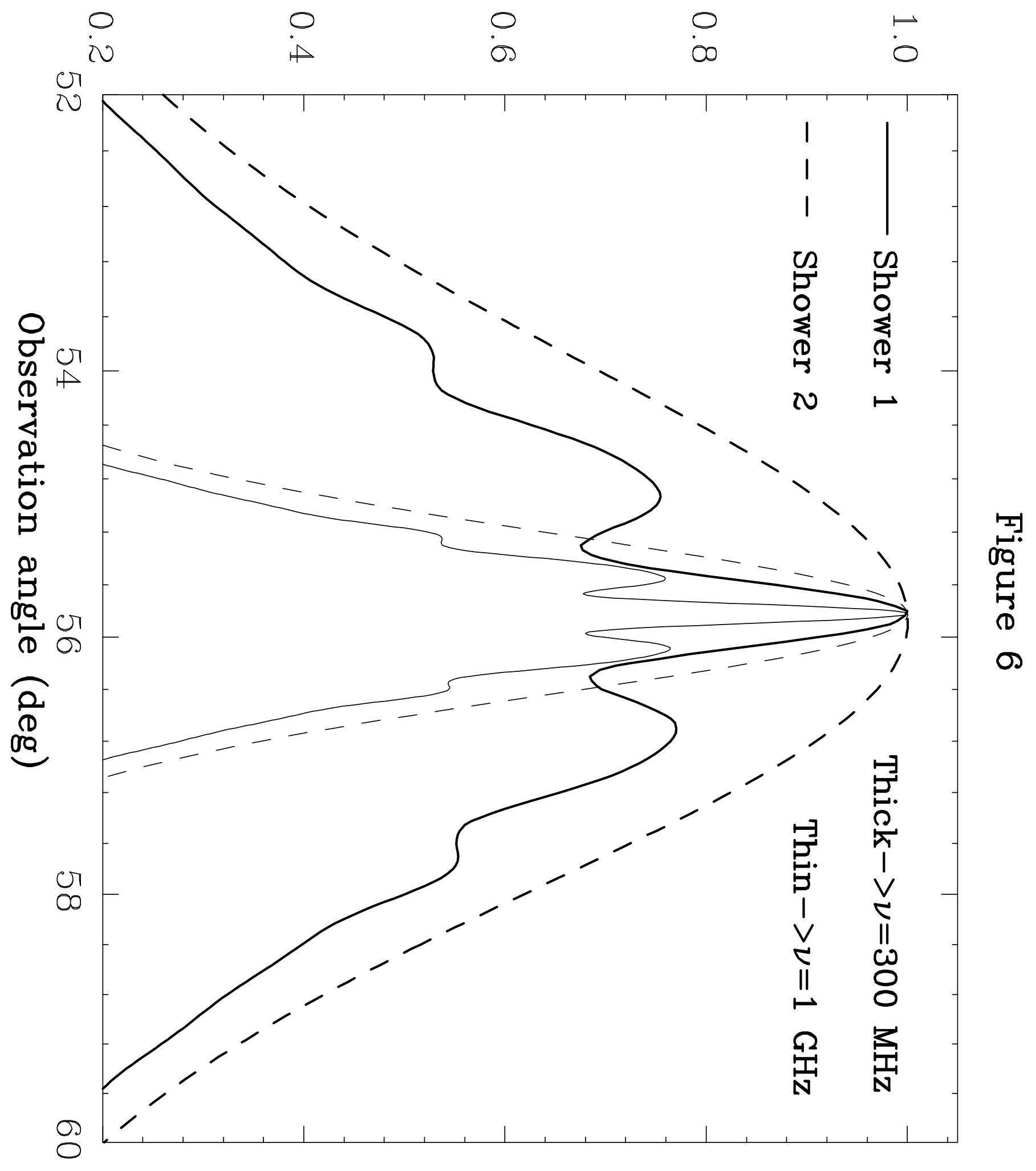




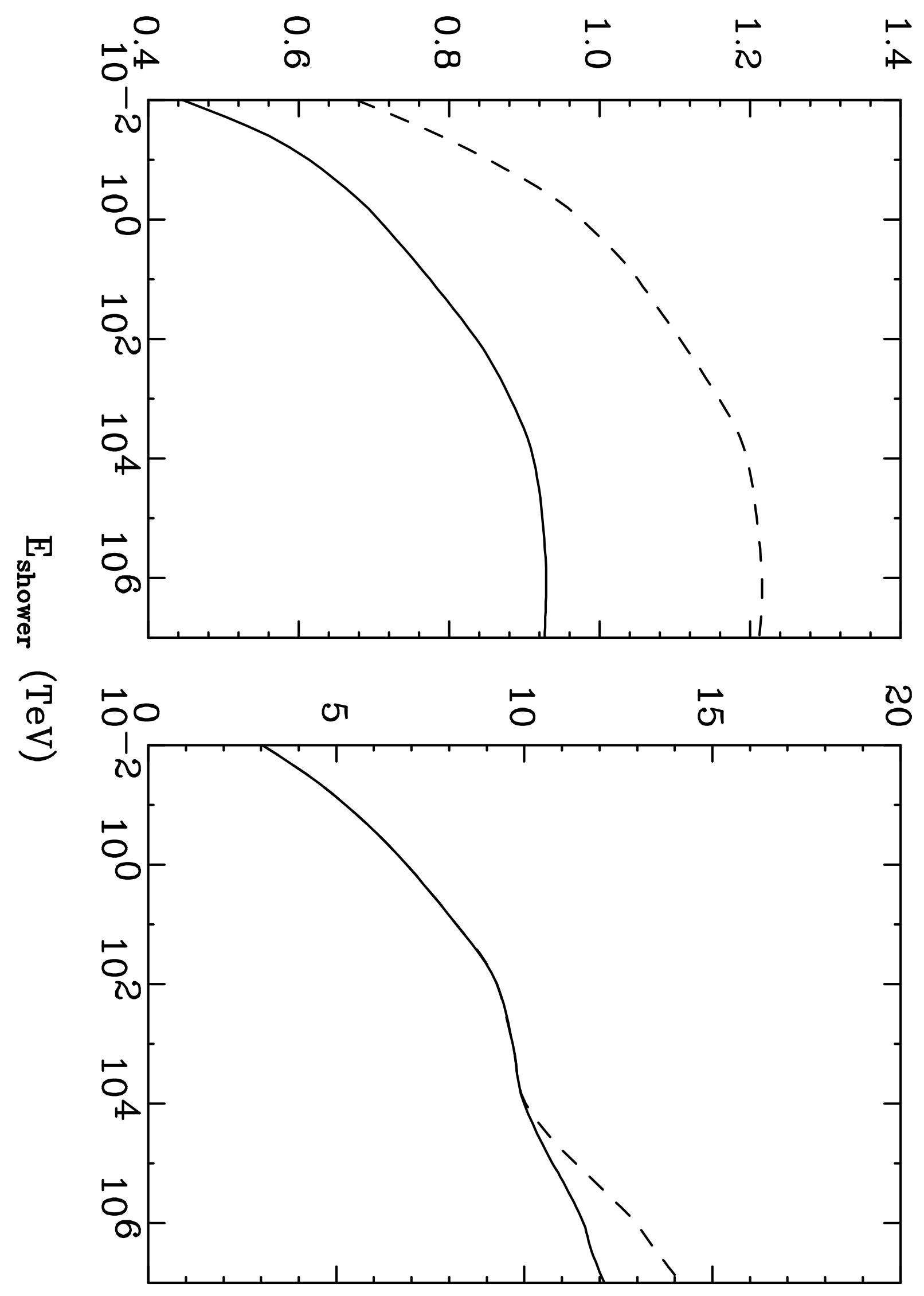

• 


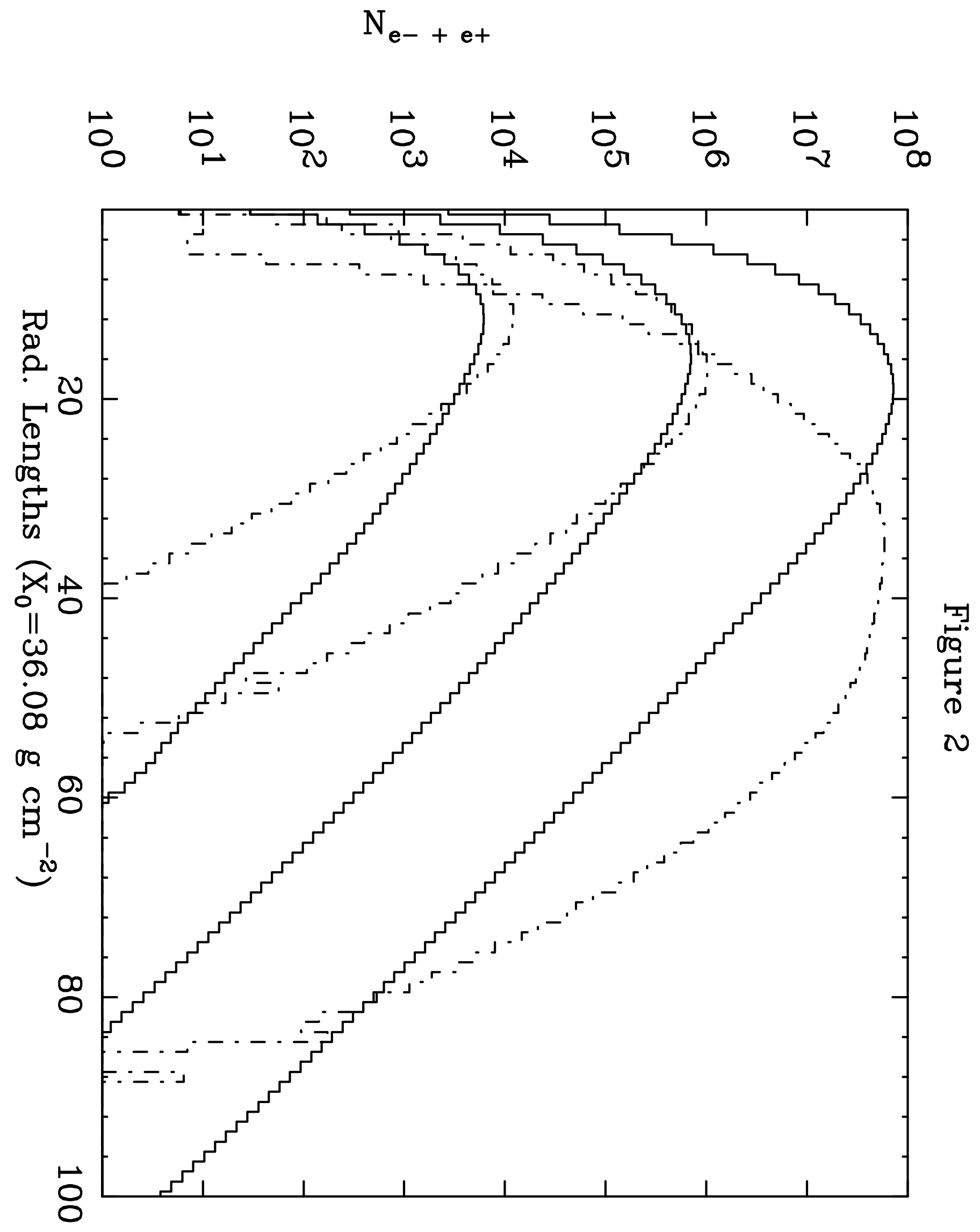




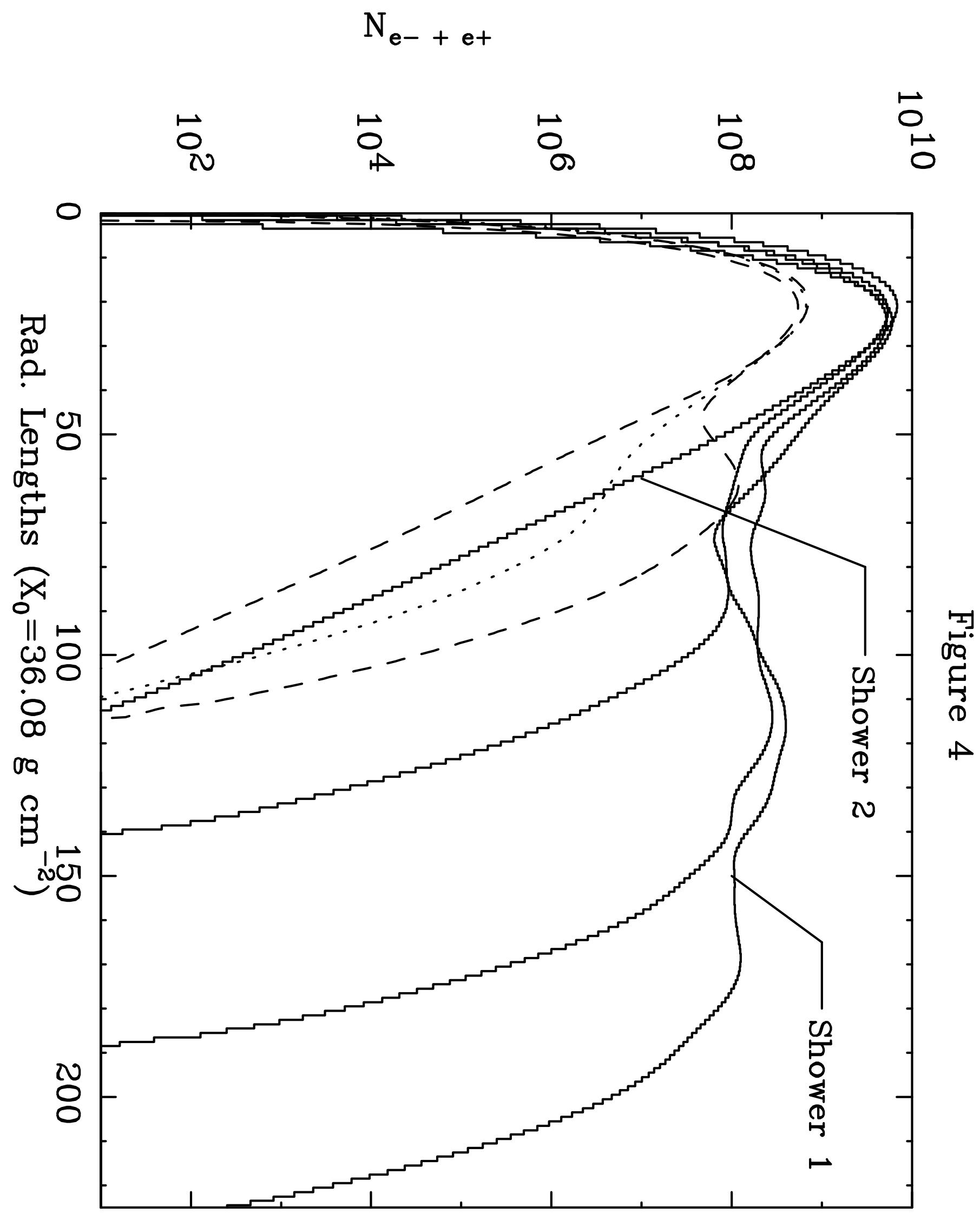


Avrg. number of $\pi^{0}$ per collision

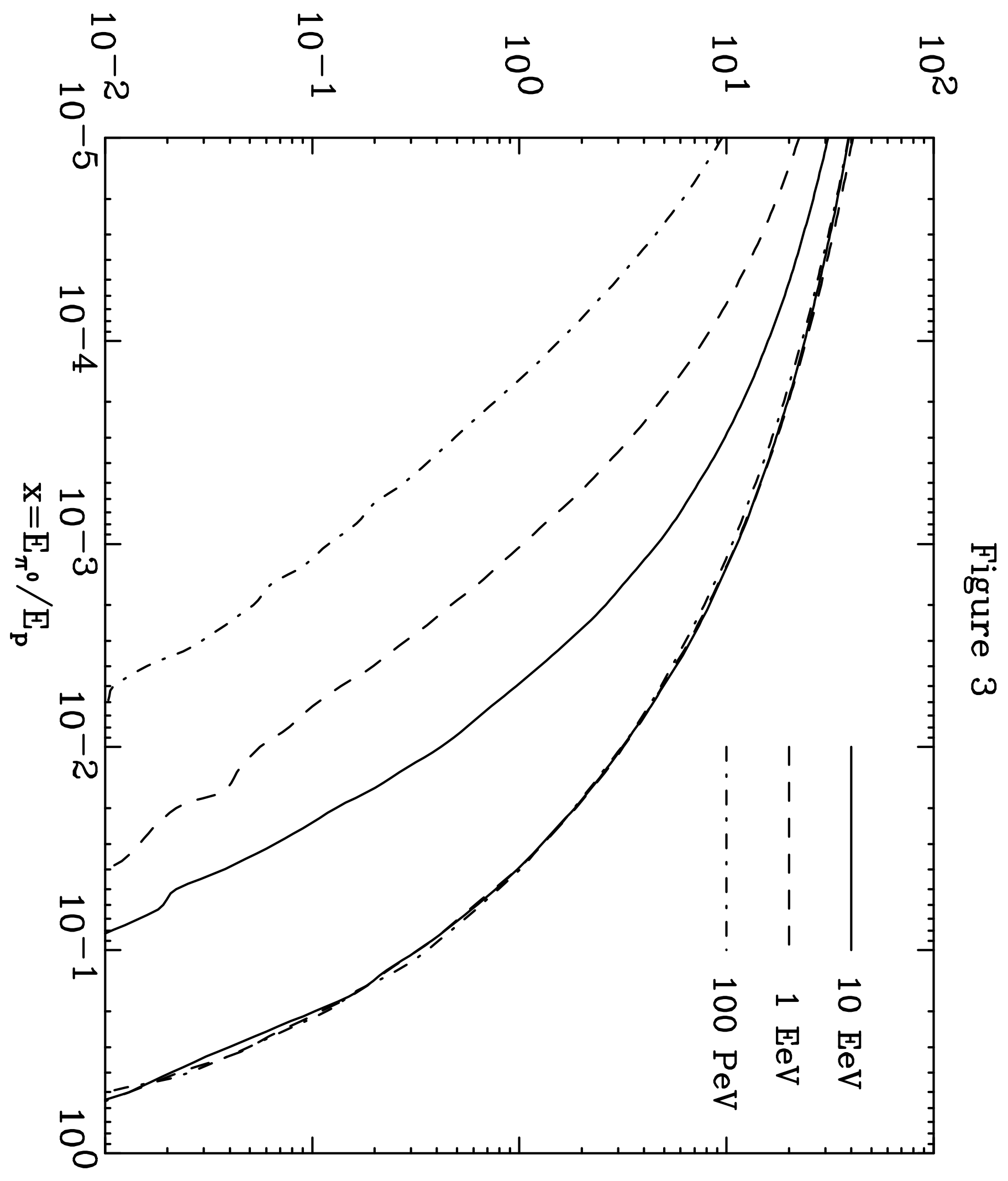

